\title{
Utilization of Cyclodextrins by Trichoderma Species
}

\author{
Gyula Oros ${ }^{*}, 1$, Annamaria Jakab ${ }^{2}$ and Tibor Cserháti ${ }^{*}, 2$ \\ ${ }^{I}$ Plant Protection Institute, Hungarian Academy of Sciences, P.O. Box 102, 1525 Budapest, Hungary \\ ${ }^{2}$ Institute of Materials and Environmental Chemistry, Chemical Research Centre, Hungarian Academy of Sciences, P.O. \\ Box 17, 1525 Budapest, Hungary
}

\begin{abstract}
The growth and the development of thallus of 17 Trichoderma species have been determined by using native $\alpha$-, $\beta$ - and $\gamma$-cyclodextrins (CDs) and 8 different CD derivatives as a unique carbon source. The majority of species can use $\mathrm{CD}$ for growth, this ability was related to their intragenic taxonomic position in order of sections Trichoderma $>$ Longibrachiatum $>$ Pachybasium $>$ Saturnisporum $T$. reesei being the most and $T$. virens the least active. All CDs have been utilized but their nutritional value was significantly influenced by the chemical structure, the size of molecules had major importance than the flexibility of their rings. Among natural derivatives of CDs Trichodermas preferred the $\alpha-$ and $\gamma$-CD prior to $\beta-C D$, when carboxylation led to reduction of the biodegradation of $\alpha$ - and $\gamma$-CDs and enhanced that of $\beta$ CD. Methylation or polymerization of $\beta-\mathrm{CD}$ also modified its decomposition rate by considered Trichoderma species. As supported by applied a three-way principal components analysis (3D-PCA) the studied here Trichoderma species showed considerable deviations in their capacity to decompose CDs and the stability of CDs towards biodegradation was highly dependent on the chemical structure of CDs.
\end{abstract}

Keywords: Biodegradation, soil parasites, cyclodextrins, structure-biodegradation relationships, multivariate analysis.

\section{INTRODUCTION}

Starch and cyclodextrins (CDs) can be used for the formulation of biologically active chemicals in solid state dispersions and liquid state [1]. CDs (see Fig. 1) are cyclic oligosaccharides commonly composed of six, seven and eight $\alpha$-D-glucose units $(\alpha-, \beta$ - and $\gamma-\mathrm{CD}$, respectively) which have an overall shape reminiscent of truncated cone. On account of their relatively hydrophobic interiors, CDs have the ability to form inclusion complexes with a wide range of substrates in aqueous solution or heterogenic systems [2,3]. This property of $\mathrm{CDs}$ has led to their application in areas as varied enzyme mimics [4-6], catalysis [7-9], environmental protection [10-12], and the encapsulation of drugs [13-16] and pesticides [17,18]. Although CDs are frequently employed in various industrial processes as carriers $[19,20]$ few attention has been paid to the study of their fate In biological systems [21-23]. It has been established that they are rapidly excreted by kidney when applied intravenously, and the rate of their intracorporal degradation is very low [24]. Bacteria associated to mammals and plants, as well as members of soil microbiota can utilize CDs [25]. Trichoderma species are common in soils of temperate and tropical climatic zones [26].

These micoparasites may interfere with arbuscular mycorhyza and can cause severe losses in commercially

*Address correspondence to these authors at the (GO) Plant Protection Institute, Hungarian Academy of Sciences, P.O. Box 102, 1525 Budapest, Hungary; Tel: +36-1-4877-538; Fax: +36-1-4877-500;

E-mail: gyoros@nki.hu; (TC) Institute of Materials and Environmental Chemistry, Chemical Research Centre, Hungarian Academy of Sciences, P.O. Box 17, 1525 Budapest, Hungary; Tel: +36-1-438-1100 ext. 442; Fax: +36-1-438-1145; E-mail: tevi@chemres.hu

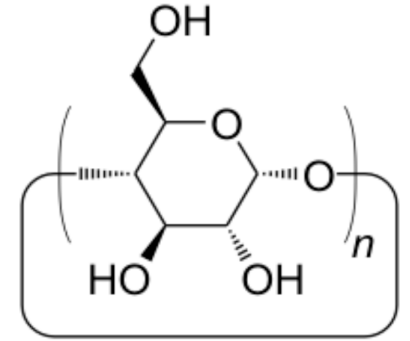

Fig. (1). Chemical structure of native cyclodextrins $(n=6,7,8$ for $\alpha-, \beta$ - and $\gamma-C D$, respectively) as the cyclic $\alpha$-D-glucose oligomers.

produced mushrooms [27]. Furthermore, they can be used for enzyme production in fermentation industry [28], for control plant diseases [29], for the promotion of plant growth [30], and for wood preservation [31].

Two-ways principal component analysis (2D-PCA), a frequently applied multivariate mathematical-statistical method, has been evolved to facilitate the extraction of maximal information from data matrices of considerable dimensions consisting numerous columns and rows [32]. The 2D-PCA allows the assessment of the correlation between the matrix elements (columns and rows) without one column or row being the dependent variable. The 2D-PCA represents the original data in reduced dimensions. It computes the relationships between the elements of the original data matrix and forms clusters of the variables according to the coefficients of correlations. The 2D-PCA has been extensively employed in diverse domains of up-to datę research. Thus, it has been used in quantitative structureactivity relationship (QSAR) investigations [33], for the assessment of molecular structure-property correlations [34], for the estimation of molecular hydrophobicity $[35,36]$, for the study of radical addition reactions [37], for quantitative 
structure-retention (QSRR) studies in gas chromatography [38], for the evaluation of structure-biodegradation relationships [39], for the classification of amino acids [40], for the assessment of solvent characteristics [41], etc.

Unfortunately, the 2D-PCA can be applied only for the determination of the similarities and differences among the columns and rows of two-dimensional data matrices and it is not suitable for the study of matrices of higher dimensions. It is possible that the information content of a three dimensional matrix composed of factors I, II, and III can be extracted by 2D-PCA. Thus, 2D-PCA has to be performed three times: (1) Factor I being the variable and factors II and III the observations, (2) Factor II being the variable and factors I and III the ob-servations, (3) Factor III being the variable and factors I and II the observations. It can be supposed that the matrices of PC loadings of the three 2DPCAs may be similar to the corresponding matrices calculated by three-way PCA (3D-PCA).

The disadvantages of 2D-PCA necessitated the development of various multiway mathematical statistical methods such as parallel factor analysis (PARAFAC) [42,43], direct fitting algorithm [44], canonical decomposition (CANDECOMP) [45], etc. The Tucker Model of 3D-PCA [46,47] and its improved version [48] have also been developed to overcome this problem. The Tucker Model has been successfully applied for the evaluation of retention data in normal phase highperformance liquid chromatography [49], the environmental studies [50], person perception analysis in psychology [51], the medium-rank second order calibration [52], and for the investigation of fermentation processes [53].

The objectives of this work were the measurement of the growth of some Trichoderma species on media containing $\mathrm{CDs}$ as unique carbon source, and the application of $3 \mathrm{D}-$ PCA for the assessment of the relationships among the capacity of Trichoderma species to utilize CDs, the elucidation of the effect of chemical structure of CDs and that of the fermentation time on their utilizability. The results may facilitate the better understanding of the biochemical and biophysical processes governing the biotransformation of $\mathrm{CDs}$ and $\mathrm{CD}$ derivatives and can find potential applications in the biotechnology of this compound class. To the best of our knowledge 3D-PCA has never been employed for the evaluation of the results of such type of utilization studies.

\section{EXPERIMENTAL}

Native $\alpha$-, $\beta$ - and $\gamma$-CDs (compounds I - III), their carboxymethylated derivatives (compounds IV - VI), DIMEB (VII, heptakis(2,6-di-O-methyl)-- $\beta$-CD), TRIMEB (VIII, heptakis(2,3,6-tri-O-methyl)- $\beta$-CD), hydroxypropylated- $\beta$-CD (IX, HP- $\beta-C D)$, water-soluble $\beta$-CD polymers: prepared by crosslinking native $\beta-C D$ molecules with ethylene glycol bis[epoxypropylether] (X, EGP) and prepared by crosslinking native $\beta$-CD molecule with buthylene glycol bis[epoxypropylether] (XI, BGP), were the gift of Dr. József Szejtli (Cyclolab Research and Development Laboratory, Budapest, Hungary). D-glucose, starch (amylum soluble) and analytical grade mineral salts (Reanal, Budapest, Hungary), pancreatic peptone (Serva, Heidelberg, Germany) and cellulose (Merck, Darmstadt,
Germany) were used as received. The agar-agar (Oxoid No. 3 , Basingstoke, UK) was purified prior to use as follows: 100 g of agar-agar was suspended in $1.0 \mathrm{~L}$ of $\mathrm{NaOH}(0.2 \mathrm{M})$ for $18 \mathrm{hrs}$, then it was washed with distilled water until neutral $\mathrm{pH}$ and dried at $55-65{ }^{\circ} \mathrm{C}$. Potato broth amended with mineral salts $\left(\mathrm{NH}_{4} \mathrm{Cl} 1.0 \mathrm{~g}, \mathrm{KH}_{2} \mathrm{PO}_{4} 1.0 \mathrm{~g}, \mathrm{~K}_{2} \mathrm{HPO}_{4} 1.0 \mathrm{~g}\right.$, $\mathrm{MgSO}_{4} \times 7 \mathrm{H}_{2} \mathrm{O} 0.5 \mathrm{~g}$ and $\mathrm{NaCl} 0.5 \mathrm{~g}$ per liter) was prepared as previously described [54] and $12 \mathrm{~g}$ of agar-agar was used for solidification.

The following strains of 17 species belonging to four sections of Trichoderma genus were used in the study of biodegradation of CDs: T. koningii Oudemans ${ }^{*}$ [signed as T1], T. aureoviride Rifai ${ }^{*}$ [T-2], T. viride Persoon ex Gray ${ }^{*}$ [T-3], $T$. atroviride Karsten [T-4]. Longibrachiatum: $T$ : ghanense Doi, Abe et Sugiyama [L-5], T. reesei Simmons* [L-6], T. pseudoningii Rifai [L-7], T. longibrachiatum Rifai $[\mathbf{L - 8}], \quad T$. parceramosum Bissett [L-9], T. citrinoviride Bissett [L-10]; Pachybasium: T. harzianum Rifai ${ }^{*}$ [P-12], T. piluliferum Webster et Rifai* ${ }^{*}$ P-13], T. polysporum (Link ex Persoon) Rifai ${ }^{*}[\mathbf{P}-14], T$. virens (Miller, Giddens, et Foster) von Arx [P-15], T. hamatum (Bonorden) Bainier ${ }^{*}$ [P-16], Hypocrea muroiana Hino et Katsumoto [P-17]; Saturnisporum: T. saturnisporum Hammill ${ }^{*}$ [S-18]. They were maintained on a potato dextrose agar (PDA) slants [54]. Species were taken from the collection of the Plant Protection Institute of Hungarian Academy of Sciences except species of T-1 to T-4, L-6 to L-8, and P-13 to P-16 which were isolated by Dr Zoltán Naár (Department of Botany, Esterházy Károly College, Eger, Hungary). Conidia used for inoculation were produced on glucose mineral agar (GMA) amended with cellulose, starch and pancreatic peptone (5.0, 10.0 and $2.0 \mathrm{~g}$ per liter, respectively). Trichoderma species were grown on media containing $5.0 \mathrm{~g}$ per liter glucose and each studied cyclodextrin as a sole carbon source. Media were dispensed in $5 \mathrm{~mm}$ deep layer into Petri dishes and were inoculated centrally with $3.0 \mu \mathrm{L}$ of spore suspension (ca $10^{6}$ conidia per $\mathrm{mL}$ ) and incubated at day/night regime at $24 \pm 2{ }^{\circ} \mathrm{C}$. Each experiment was performed in triplicate. The response of fungi to the type of carbon source was characterized by the measurement of the linear growth of hypha (colony diameter in $\mathrm{mm}$ ) at each day up till seven days. The actual colony diameters were expressed in the percent of the colony diameter of $T$. viride grown on GMA at sixth day.

The Tucker Model of 3D-PCA has been employed for the calculations [46-53]. The data matrix consisted of the 17 strains of Trichoderma (factor A), the $11 \mathrm{CDs}$ and $\mathrm{CD}$ derivatives (factor B) and the 6 incubation time (factor C) compiled in Tables 1-6 (3-way array with dimensions $17,11,6)$. In order to extract the maximal information from the original data matrix, the calculation was firstly performed on the component matrixes of the largest possible dimensions $(16,10,5)$. The component matrices explaining minimally $1.0 \%$ of the total variance (in this case $3,3,2$ ) were selected and the calculation was performed again on this reduced component matrix. The similarities and dissimilarities among the capacity of strains to use CDs as unique carbon source, among CDs to be decomposed by the Trichoderma species and incubation times were visualized by plotting the first column of the corresponding component matrices $v s$ the second column. The reality of this plotting method was justified the high ratio of variance explained by the first two columns. 
Table 1. The 24 Hours Linear Growth of Trichoderma Species on Media Containing CDs and CD Derivatives as Unique Carbon Sources

\begin{tabular}{|c|c|c|c|c|c|c|c|c|c|c|c|}
\hline Species & \multicolumn{11}{|c|}{ Cycl odextrins and Cyclodextrin Derivatives } \\
\hline T-2 & 0 & 0 & 0 & 0 & 0 & 0 & 0 & 0 & 0 & 0 & 0 \\
\hline $\mathrm{T}-3$ & 0 & 0 & 0 & 0 & 0 & 0 & 0 & 0 & 0 & 0 & 0 \\
\hline L-6 & 0 & 0 & 0 & 0 & 0 & 0 & 0 & 0 & 0 & 0 & 0 \\
\hline $\mathrm{L}-7$ & 0 & 0 & 0 & 0 & 0 & 0 & 0 & 0 & 0 & 0 & 0 \\
\hline L-8 & 1 & 0 & 0 & 0 & 0 & 0 & 0 & 0 & 0 & 0 & 0 \\
\hline L-9 & 0 & 0 & 0 & 0 & 0 & 0 & 0 & 0 & 0 & 0 & 0 \\
\hline P-14 & 0 & 0 & 0 & 0 & 0 & 0 & 0 & 0 & 0 & 0 & 0 \\
\hline P-15 & 0 & 0 & 2 & 0 & 1 & 0 & 0 & 0 & 0 & 0 & 0 \\
\hline P-16 & 0 & 0 & 1 & 0 & 0 & 1 & 0 & 0 & 0 & 0 & 4 \\
\hline P-17 & 0 & 0 & 0 & 0 & 0 & 0 & 0 & 0 & 0 & 0 & 0 \\
\hline S-18 & 0 & 0 & 0 & 0 & 0 & 0 & 0 & 0 & 0 & 0 & 0 \\
\hline
\end{tabular}

Note: Average diameters determined after 24 hours of incubation in percent of the diameter of T. viride grown on the GMA at sixth day. For abbreviations see Experimental. Roman numbers refer to $\mathrm{CDs}$ and $\mathrm{CD}$ derivatives.

Table 2. The 48 Hours Linear Growth of Trichoderma Species on Media Containing CDs and CD Derivatives as Unique Carbon Sources

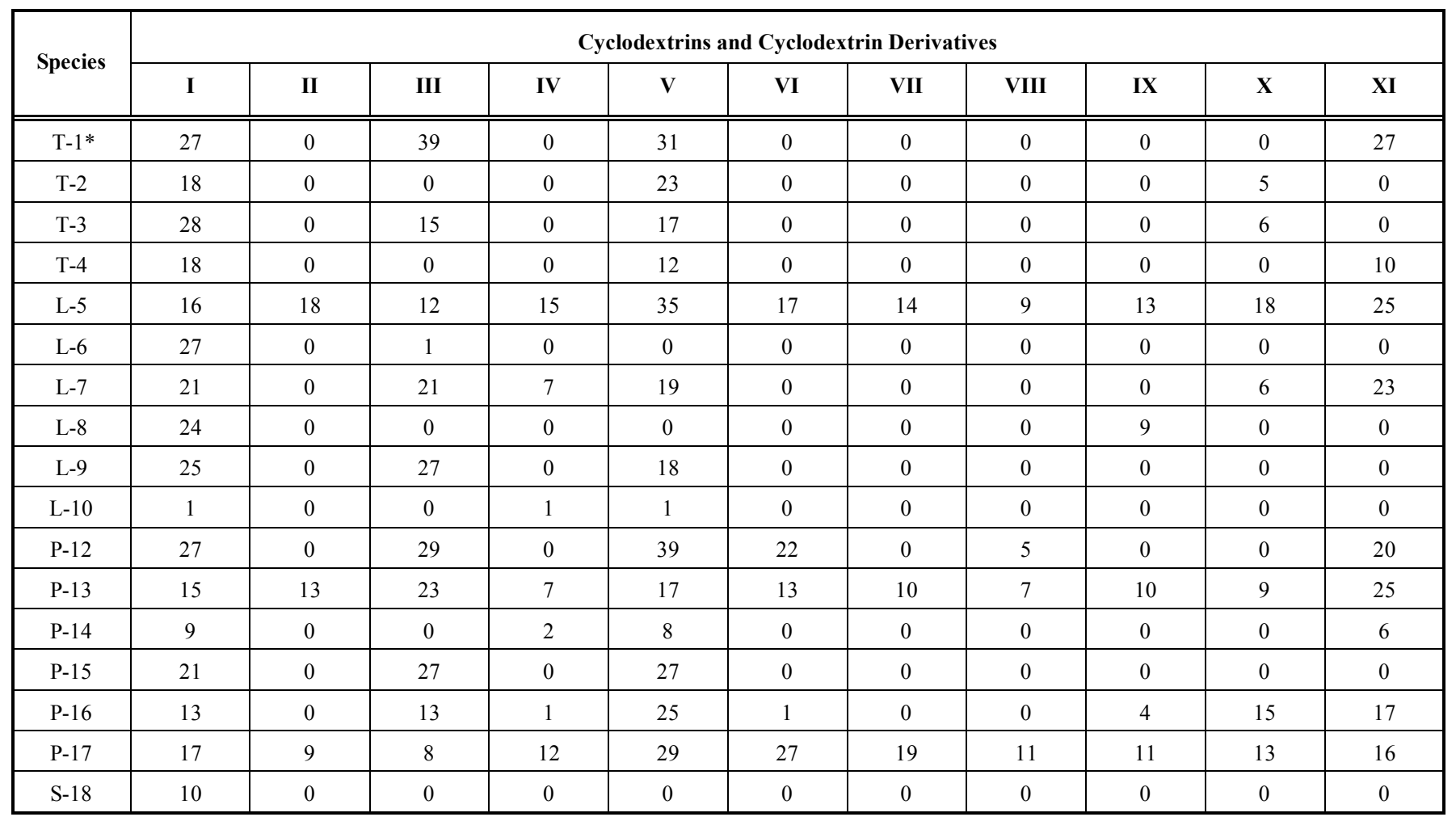


Table 3. The 72 Hours Linear Growth of Trichoderma Species on Media Containing CDs and CD Derivatives as Unique Carbon Sources

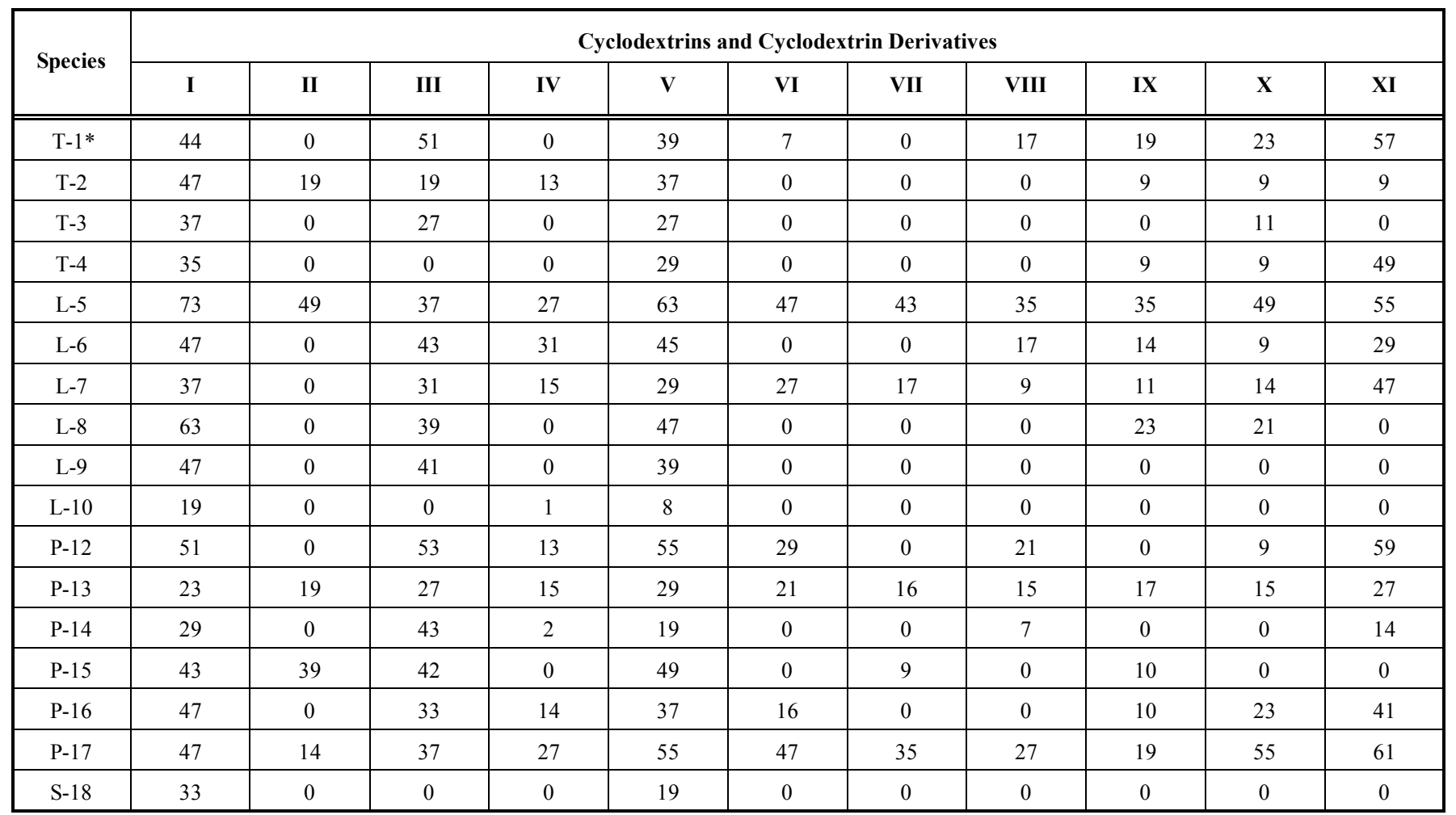

Note: Average diameters determined after 24 hours of incubation in percent of the diameter of $T$. viride grown on the GMA at sixth day. For abbreviations see Experimental. Roman numbers refer to $\mathrm{CDs}$ and $\mathrm{CD}$ derivatives.

Table 4. The 96 Hours Linear Growth of Trichoderma Species on Media Containing CDs and CD Derivatives as Unique Carbon Sources

\begin{tabular}{|c|c|c|c|c|c|c|c|c|c|c|c|}
\hline Species & \multicolumn{11}{|c|}{ Cyclodextrins and Cyclodextrin Derivatives } \\
\hline $\mathrm{T}-2$ & 69 & 19 & 56 & 35 & 39 & 0 & 0 & 9 & 19 & 24 & 53 \\
\hline $\mathrm{T}-3$ & 49 & 0 & 35 & 30 & 39 & 19 & 21 & 0 & 20 & 24 & 0 \\
\hline L-6 & 63 & 0 & 75 & 45 & 73 & 0 & 0 & 25 & 29 & 21 & 67 \\
\hline $\mathrm{L}-7$ & 49 & 0 & 47 & 25 & 45 & 43 & 29 & 23 & 29 & 29 & 69 \\
\hline L-8 & 69 & 0 & 47 & 37 & 57 & 0 & 9 & 0 & 49 & 39 & 61 \\
\hline L-9 & 63 & 0 & 53 & 0 & 39 & 0 & 0 & 0 & 0 & 0 & 0 \\
\hline P-14 & 33 & 43 & 53 & 7 & 35 & 0 & 9 & 14 & 23 & 19 & 29 \\
\hline P-15 & 65 & 39 & 42 & 0 & 59 & 0 & 0 & 0 & 23 & 0 & 53 \\
\hline P-16 & 59 & 0 & 39 & 33 & 39 & 30 & 0 & 0 & 21 & 39 & 55 \\
\hline P-17 & 63 & 16 & 77 & 37 & 57 & 71 & 79 & 45 & 75 & 83 & 95 \\
\hline S-18 & 47 & 53 & 6 & 0 & 35 & 0 & 0 & 0 & 0 & 18 & 0 \\
\hline
\end{tabular}

Note: Average diameters determined after 24 hours of incubation in percent of the diameter of T. viride grown on the GMA at sixth day. For abbreviations see Experimental. Roman numbers refer to $\mathrm{CDs}$ and $\mathrm{CD}$ derivatives. 
Table 5. The 120 Hours Linear Growth of Trichoderma Species on Media Containing CDs and CD Derivatives as Unique Carbon Sources

\begin{tabular}{|c|c|c|c|c|c|c|c|c|c|c|c|}
\hline \multirow{2}{*}{ Species } & \multicolumn{11}{|c|}{ Cyclodextrins and Cyclodextrin Derivatives } \\
\hline & $\mathbf{I}$ & II & III & IV & $\mathbf{V}$ & VI & VII & VIII & IX & $\mathbf{X}$ & XI \\
\hline $\mathrm{T}-1^{*}$ & 59 & 0 & 51 & 43 & 48 & 19 & 27 & 31 & 40 & 55 & 64 \\
\hline $\mathrm{T}-2$ & 69 & 29 & 56 & 39 & 58 & 12 & 25 & 31 & 39 & 43 & 76 \\
\hline $\mathrm{T}-3$ & 49 & 0 & 41 & 40 & 42 & 21 & 37 & 13 & 47 & 33 & 4 \\
\hline $\mathrm{T}-4$ & 54 & 39 & 49 & 37 & 49 & 9 & 0 & 25 & 40 & 45 & 49 \\
\hline L-5 & 99 & 89 & 99 & 49 & 99 & 70 & 59 & 63 & 79 & 90 & 95 \\
\hline L-6 & 63 & 22 & 75 & 49 & 73 & 37 & 0 & 34 & 57 & 55 & 69 \\
\hline L-7 & 49 & 0 & 47 & 33 & 49 & 45 & 36 & 37 & 39 & 45 & 74 \\
\hline L-8 & 73 & 49 & 46 & 47 & 57 & 8 & 33 & 51 & 51 & 47 & 69 \\
\hline L-9 & 64 & 0 & 59 & 20 & 55 & 43 & 0 & 9 & 0 & 13 & 2 \\
\hline L-10 & 46 & 45 & 19 & 3 & 59 & 4 & 0 & 10 & 0 & 39 & 29 \\
\hline P-12 & 73 & 49 & 63 & 56 & 59 & 49 & 37 & 39 & 37 & 43 & 77 \\
\hline P-13 & 41 & 53 & 49 & 49 & 69 & 59 & 44 & 39 & 47 & 51 & 78 \\
\hline P-14 & 39 & 56 & 57 & 14 & 42 & 14 & 24 & 38 & 31 & 37 & 43 \\
\hline $\mathrm{P}-15$ & 66 & 50 & 45 & 20 & 64 & 14 & 3 & 28 & 39 & 7 & 60 \\
\hline P-16 & 59 & 45 & 39 & 33 & 39 & 31 & 0 & 24 & 49 & 49 & 56 \\
\hline P-17 & 75 & 51 & 94 & 50 & 69 & 80 & 87 & 57 & 87 & 89 & 100 \\
\hline S-18 & 48 & 53 & 39 & 13 & 49 & 14 & 0 & 25 & 27 & 37 & 34 \\
\hline
\end{tabular}

Note: Average diameters determined after 24 hours of incubation in percent of the diameter of T. viride grown on the GMA at sixth day. For abbreviations see Experimental. Roman numbers refer to $\mathrm{CDs}$ and $\mathrm{CD}$ derivatives.

Table 6. The 144 Hours Linear Growth of Trichoderma Species on Media Containing CDs and CD Derivatives as Unique Carbon Sources

\begin{tabular}{|c|c|c|c|c|c|c|c|c|c|c|c|}
\hline Species & \multicolumn{11}{|c|}{ Cyclodextrins and Cyclodextrin Derivatives } \\
\hline $\mathrm{T}-2$ & 69 & 67 & 56 & 39 & 59 & 39 & 47 & 47 & 53 & 45 & 78 \\
\hline $\mathrm{T}-3$ & 64 & 0 & 59 & 9 & 49 & 37 & 41 & 40 & 61 & 37 & 5 \\
\hline L-6 & 63 & 49 & 75 & 61 & 73 & 40 & 35 & 43 & 59 & 55 & 87 \\
\hline $\mathrm{L}-7$ & 59 & 0 & 59 & 47 & 49 & 53 & 49 & 43 & 49 & 55 & 78 \\
\hline L-8 & 73 & 67 & 49 & 63 & 64 & 14 & 55 & 55 & 55 & 57 & 69 \\
\hline L-9 & 69 & 0 & 59 & 59 & 74 & 43 & 0 & 35 & 0 & 27 & 0 \\
\hline P-14 & 47 & 69 & 65 & 21 & 49 & 43 & 35 & 41 & 41 & 43 & 49 \\
\hline $\mathrm{P}-15$ & 69 & 65 & 46 & 47 & 69 & 39 & 55 & 35 & 50 & 47 & 61 \\
\hline P-16 & 59 & 63 & 41 & 43 & 41 & 55 & 44 & 51 & 61 & 50 & 79 \\
\hline $\mathrm{P}-17$ & 85 & 68 & 100 & 60 & 100 & 100 & 99 & 73 & 100 & 100 & 100 \\
\hline S-18 & 51 & 63 & 49 & 2 & 63 & 39 & 0 & 39 & 43 & 49 & 81 \\
\hline
\end{tabular}

Note: Average diameters determined after 24 hours of incubation in percent of the diameter of T. viride grown on the GMA at sixth day. For abbreviations see Experimental. Roman numbers refer to $\mathrm{CDs}$ and $\mathrm{CD}$ derivatives. 
The effect of the reduction of the dimensions of component matrices was assessed by calculating linear correlations between the first and second columns of component matrices calculated from the largest and reduced component matrices. Software for the 3D-PCA was taken from N-Way Toobox for Matlab v.7.3 developed by Dr Claus A. Andersson and Dr Rasmus Bro from Faculty of Life Sciences, University of Kopenhagen, Frederiksberg C, Denmark (see web page: http://newton.mli.kvl.dk/algorit hms). Linear regressions were calculated by the "Drugidea" software purchased from CompuDrug Ltd (Budapest, Hungary).

\section{RESULTS AND DISCUSSION}

The values of linear growth of Trichoderma species on CDs and CD derivatives at various incubation lengths are compiled in Tables 1-6. The data clearly illustrate that the nutritive values of CDs were in each instance lower than that of glucose demonstrating that CDs are less appropriate carbon sources for Trichoderma species than glucose. It can be further observed that the nutritive values of CDs differ considerably from each other suggesting that the structure of CDs influences the biodegradation rate.

The main parameters of the 3D-PCAs calculated form the largest and reduced matrices are compiled in Table 7. 3D-PCA using component matrix 16, 10, 5 reached the convergence criteria after the third iteration step, the total variance explained being $99.77 \%$. The data demonstrate that the first factor explains the majority of variance $(87.15 \%)$ indicating the fundamental similarities between the Trichoderma species, CDs and incubation times. Interestingly, the marked reduction in the dimensions of component matrix resulted in the slight increase in the number of iterations and in a negligible loss of variance explained (6.22) proving that the reduced component matrix can also be used for the extraction of similar amount of Information from the original data matrix. The comparison of the results of the two calculations further shows that this empirical method can be successfully used for the reduction of dimensionality of the component matrix.

The similarities and dissimilarities between the Trichoderma species simultaneously taking into consideration each $\mathrm{CD}$ and each incubation time (plot of the first and second columns of factor A calculated from the reduced matrix) are shown in Fig. (2). The data demonstrate that Trichoderma species do not form well-defined clusters according to their intrageneric taxonomical position that is the intrageneric taxonomical position do not exert a considerable influence on their capacity to utilize CDs and CD derivatives.

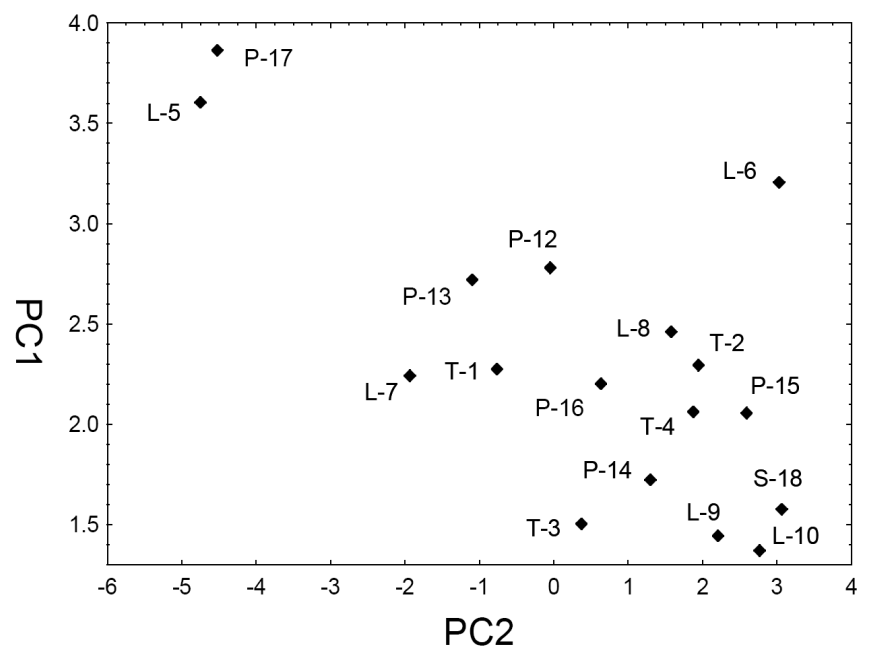

Fig. (2). Similarities and dissimilarities between the studied Trichoderma species simultaneously taking into consideration each of applied cyclodextrin and each studied incubation time (plot of the first and second columns of factor A calculated from the reduced matrix). Symbols refer to Trichoderma species as given in Experimental and Tables 1-6.

Table 7. Main Parameters of the Three-Dimensional PCAs Using the Largest $(16,10,5)$ and the Reduced $(3,3,2)$ Component Matrices

\begin{tabular}{|c|c|c|c|c|c|}
\hline \multicolumn{4}{|c|}{ Dimensions of Component Matrices } & No. Iterations & \multirow{2}{*}{$\begin{array}{c}\text { Total Variance Explained (\%) } \\
99.77\end{array}$} \\
\hline & 16 , & 10 , & 5 & \multirow[t]{7}{*}{3} & \\
\hline No & \multicolumn{3}{|c|}{ No of Factors of Component Matrices } & & Variance Explained of the Total Variance Explained (\%) \\
\hline & A & B & $\mathrm{C}$ & & \\
\hline 1 & 1 & 1 & 1 & & 87.15 \\
\hline 2 & 1 & 2 & 2 & & 1.36 \\
\hline 3 & 2 & 1 & 2 & & 1.15 \\
\hline 4 & 3 & 3 & 1 & & 0.99 \\
\hline \multicolumn{4}{|c|}{ Dimensions of Component Matrices } & No. iterations & Total Variance Explained (\%) \\
\hline & 3 & 3 , & 2 & \multirow[t]{7}{*}{4} & 92.95 \\
\hline No & \multicolumn{3}{|c|}{ No of Factors of Component Matrice } & & Variance Explained of the Total Variance Explained (\%) \\
\hline & A & B & $\mathrm{C}$ & & \\
\hline 1 & 1 & 1 & 1 & & 93.55 \\
\hline 2 & 1 & 2 & 2 & & 1.49 \\
\hline 3 & 2 & 1 & 2 & & 1.21 \\
\hline 4 & 3 & 3 & 1 & & 1.02 \\
\hline
\end{tabular}


CDs and CD derivatives do not form clear-cut clusters on the plot of the first and second columns of factor B calculated from the reduced matrix taking into consideration simultaneously each Trichoderma species and incubation time (Fig. 3). This findings, contrary to previously reported for Xanthomonas bacterial plant species [54], suggests that the dimensions of the CD cavity, the character of substituents and the mode of polymerisation have a similar impact on their sensitivity towards biodegradation by Trichoderma species.

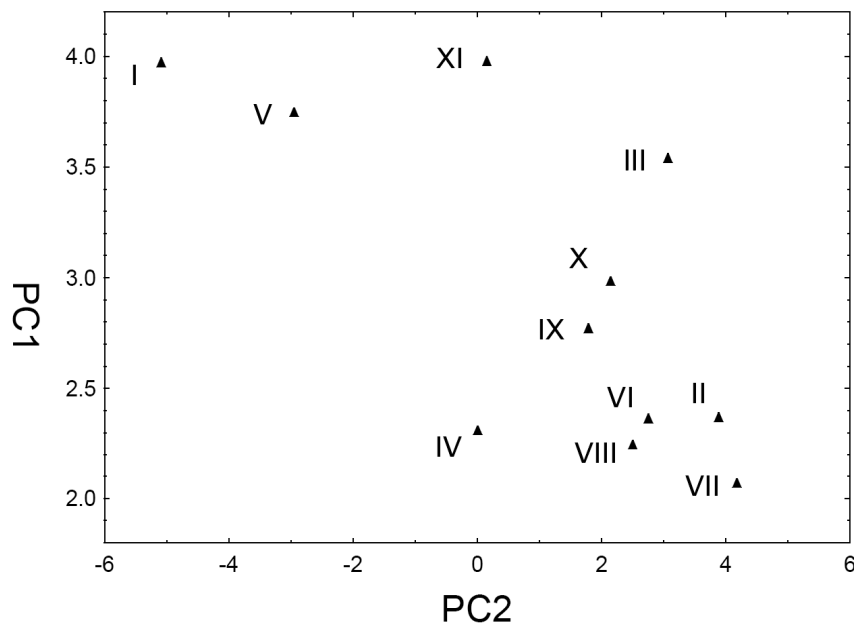

Fig. (3). Distribution of cyclodextrins and cyclodextrin derivatives simultaneously taking into consideration each Trichoderma species and applied incubation time (plot of the first and second columns of factor B calculated from the reduced matrix). Symbols refer to studied CDs and $\mathrm{CD}$ derivatives: I: $\alpha-\mathrm{CD}$, II: CM- $\alpha-\mathrm{CD}, \beta-\mathrm{CD}$, III: $\gamma-C D$, IV: CM- $\alpha-C D, \mathbf{V}:$ CM- $\beta-C D$, VI: CM- $\gamma$-CD, VII: DIMEB, VIII: TRIMEB, IX: HP- $\beta-C D, \mathbf{X}$ : EGP, XI: BGP.

Incubation times show marked deviations simultaneously taking into consideration each Trichoderma species and each $\mathrm{CD}$ and $\mathrm{CD}$ derivative (plot of the first and second columns of factor $\mathrm{C}$ calculated from the reduced matrix) as demonstrated in Fig. (4). This scattering can be tentatively explained by the supposition that the differences among the biodegradation of CDs is higher at higher incubation time.

Highly significant linear correlations were found between the first and second columns of factors A, B and C calculated from the largest and reduced component matrices. The coefficients of correlation were 0.9999 (column 1 of factor A), 0.9967 (column 2 of factor A), 0.9998 (column 1 of factor B), 0.9958 (column 2 of factor B), 0.9999 (column 1 of factor C), and 0.9988 (column 2 of factor C). The high significance level of correlations proves that similarly to the total variance explained the reduction of the dimensionality of component matrices does not influence the similarities and dissimilarities among the matrix elements.

\section{CONCLUSIONS}

It has been established that Trichoderma species are potent deteriorators of native CDs and their derivatives and they may promote the elimination of this class of compounds from any organic and inorganic matrices. As the decomposition rate considerably depends on both the type of Trichoderma species and the chemical structure of CDs an

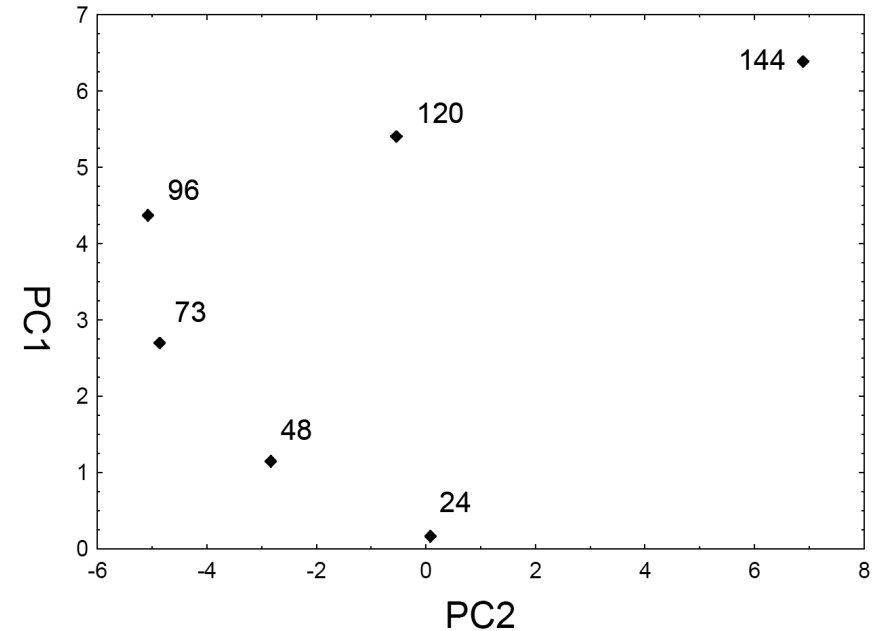

Fig. (4). Classification of incubation times simultaneously taking into consideration each studied Trichoderma species and each CDs (plot of the first and second columns of factor $\mathrm{C}$ calculated from the reduced matrix). Numbers refer to studied incubation time in hours.

adequate selection of species for the decomposition of a given $\mathrm{CD}$ is necessary. Our results indicate that mould Trichodermas preferred the $\alpha$ - and $\gamma-\mathrm{CD}$ prior to $\beta-\mathrm{CD}$, when carboxylation led to reduction of the biodegradation of $\alpha$ - and $\gamma$-CDs and enhanced that of $\beta$-CD. Methylation or polymerization of $\beta-\mathrm{CD}$ also modified it decomposition rate by considered Trichoderma species. These conclusions are dissimilar to previously reported degradation pattern observed in the case of Xanthomonas species which preferred $\gamma$-CD utilization [54]. In addition, the results of presented work indicate that 3D-PCA procedure can be successfully employed for the study of the microbiological decomposition of $\mathrm{CDs}$ and $\mathrm{CD}$ derivatives. The method outlined above can be used for the reduction of the dimensionality of component matrices exerting a negligible effect on the variance explained and on the similarities and dissimilarities among the elements of the original matrix. We have to stress that the deductions discussed above are not based on correct mathematical treatments, they are empirical ones and their validity for another data matrices has not been verified. Therefore, the extrapolation of the conclusions above to other data matrices may result in serious misinterpretation of the results.

\section{ACKNOWLEDGEMENTS}

The authors express to their gratitude Dr. József Szejtli (Cyclolab, Budapest, Hungary) and Dr. Zoltan Naár (Esterházy Károly College, Eger, Hungary) for supplying the cyclodextrins and Trichoderma isolates, respectively.

\section{REFERENCES}

[1] Schierbaum, F.; Vorwerg, W. Starches and their hydrolysis products as pharmaceutical adjuvants. Part III: Survey on preparation and characterization of active agents and their carriers. Starch-Stärke 1997, 49,30 .

[2] Fenyvesi, E.; Nagai, T.; Antal, B.; Zsadon, B.; Szejtli, J. Evaluation of cyclodextrin polymer as s disintegrating agent. J. Incl. Phenom. 1984, 2, 645.

[3] McCray, J.E.; Brusseau, M.L. Cyclodextrin-enhanced in-situ flushing of multiple-component immiscible organic liquid contamination at the field scale: mass removal effectiveness. Environ. Sci. Technol. 1998, 32,1285 . 
[4] Breslow, R.; Anslyn, E.; Huang, D.L. Ribonuclease mimics. Tetrahedron, 1991, 47, 2365.

[5] Yuan, D.Q.; Dong, S.D.; Breslow, R. Cyclodextrin-based class-I aldolase enzyme mimics to catalyze crossed aldol condensations. Tetrahedron Lett., 1998, 39, 7673.

[6] Lee, W.S.; Ueno, A. A new feature of bifunctional catalysis. Cyclodextrins bearing two imidazole moieties as hydrolysis enzyme model. Chem. Lett., 2000, 3, 258.

[7] Komiyama, M. Cyclic oligomers as highly selective catalysts. Progr. Polym. Sci., 1993, 18, 871.

[8] Jarho, P.; Van der Velde, D.; Stella, V.J. Cyclodextrin-catalyzed deacetylation of spironolactone is $\mathrm{pH}$ and cyclodextrin dependent. $J$. Pharm. Sci., 2000, 89, 241.

[9] Chou, D.T.H.; Zhu, J.; Huang, X.C.; Bennet, A.J. Cyclodextrin catalysis of the $\mathrm{pH}$-independent hydrolyses of acetals. J. Chem. Soc. Perkin Trans., 2001, 2, 83.

[10] Bardi, L.; Mattei, A.; Steffan, S.; Marzona, M. Hydrocarbon degradation by a soil microbial population with $\beta$-cyclodextrin as surfactant to enhance bioavailability. Enzyme Microb. Techn., 2000, 27, 709.

[11] Fava, F.; Di Gioia, D.; Marchetti, L. Cyclodextrin effects on the ex-situ bioremediation of a chronically polychlorobiphenyl-contaminated soil. Biotechnol. Bioeng., 1998, 58, 345.

[12] Wang, J.M.; Marlowe, E.M.; Miller-Maier, R.M.; Brusseau, M.L. Cyclodextrin-enhanced biodegradation of phenanthrene. Environ. Sci. Technol., 1998, 32, 1907.

[13] Loftsson, T.; Brewster, M.E. Pharmaceutical applications of cyclodextrins. Drug solubilization and stabilization. J. Pharm. Sci., 1996, 85,1017

[14] Thompson, D.O. Cyclodextrins-enabling excipients: their present and future use in pharmaceuticals. Crit. Rev. Ther. Drug Carr. Syst., 1997, 14,1 .

[15] Merkus, F.W.H.M.; Verhoef, J.C.; Marttin, E.; Romeijn, S.G.; Van der Kuy, P.H.M.; Hermens, W.A.J.J.; Schipper N.G.M. Cyclodextrins in nasal drug delivery. Adv. Drug Deliv. Rev., 1999, 36, 41.

[16] Loftsson, T.; Jarvinen, T. Cyclodextrins in ophthalmic drug delivery. Adv. Drug. Deliv. Syst., 1999, 36, 59.

[17] Cserháti, T.; Szente, L.; Szejtli, J. Complex-forming eluent additives in reversed-phase thin layer chromatography of methylated betacyclodextrins. J. High Resol. Chromatogr., 1984, 7, 635.

[18] Szejtli, J. Utilization of cyclodextrins in industrial products and processes. J. Mater. Chem., 1997, 7, 575.

[19] Duchene, D.; Wouessidjewe, D.; Ponchel, G. Cyclodextrins and carrier systems. J. Control. Rel., 1999, 62, 263.

[20] Redenti, E.; Szente, L.; Szejtli, J. Drug/cyclodextrin/hydroxy acid multicomponent systems. Properties and pharmaceutical applications. $J$. Pharm. Sci., 2000, 89, 1.

[21] Szejtli, J.; Gerloczy, A.; Fonagy, A. Intestinal absorption of ${ }^{14} \mathrm{C}$-labelled $\beta$-cyclodextrin in rats. Drug Res., 1980, 30, 808.

[22] Gerloczy, A.; Fonagy, A.; Szejtli, J. Absorption and metabolism of beta-cyclodextrin by rats. Szejtli, J.; Ed.; Proc. First Int. Symp. Cyclodextrins, Reidel, Dordrecht: The Netherlands, 1981, p. 101.

[23] Szabó, P.; Ferenczy, T.; Serfözö, J.; Liptak, A. Absorption and elimination of cyclodextrin derivatives by rabbits and rats. Szejtli, J.; Ed.; Proc. First Int. Symp. Cyclodextrins, Reidel, Dordrecht: The Netherlands, 1981, p. 115.

[24] Kubota, Y.; Sanbe, H.; Koizumi, K. Absorption, distribution and excretion of galactosyl- $\beta$-cyclodextrin and mannosyl- $\beta$-cyclodextrin in rats. Biol. Pharm. Bull., 2000, 23, 472.

[25] Oros, G.; Cserháti, T.; Fenyvesi, E.; Szejtli, J. Mcrobial decomposition of some cyclodextrin derivatives by bacteria associated with plants. Int. Biodeterioration, 1990, 26, 33.

[26] Papavizas, G.C. Trichoderma and gliocladium: biology, ecology, and potential for biocontrol. Ann. Rev. Phytopathol., 1985, 23, 923.

[27] Samuels, G.J. Trichoderma: a review of biology and systematics of the genus. Mycol. Res., 1966, 100, 923.

[28] Wood, T.M. Cellulolytic enzyme system of Trichoderma koningii. Separation of components attacking native cotton. Biochem. J., 1968, $109,217$.
[29] Naár, Z.; Kecskés, M. Factors influencing the competitive saprophytic ability of Trichoderma species. Microbiol. Res., 1998, 153, 119.

[30] Ousley, M.A.; Lynch, J.M.; Whipps, J.M. Potential of trichoderma spp. as consistent plant growth stimultors. Biol. Fert. Soil, 1994, 17, 85.

[31] Highley, T.L. Control of wood decay by Trichoderma (Gliocladium) virens. I. Antagonistic properties. Mater. Organ, 1997, 31, 79.

[32] Mardia, K.V.; Kent, J.T.; Bibby, J.M. Multivariate Analysis; Academic Press: London, 1979, p. 213.

[33] Drew, M.G.B.; Wilden, G.R.H.; Spillane, W.J.; Walsh, R.M.; Ryder C.A.; Simmie, J.M. Quantitative structure-activity relationship studies of sulfamates $\mathrm{RNHSO}_{3} \mathrm{Na}$ : distinction between sweet, sweet-bitter, and bitter molecules. J. Agric. Food Chem., 1998, 46, 3016.

[34] Seybold, P.G. Explorations of molecular structure-property relationships. SAR QSAR Environ. Res., 1999, 10, 101.

[35] Sarbu, C.; Todor, S. Evaluation of lipophilicity by principal components analysis. J. Planar Chromatogr. Mod. TLC, 1998, 11, 123.

[36] Mannhold, R.; Cruciani, G.; Dross, K.; Rekker, R. Multivariate analysis of experimental and computational descriptors of molecular lipophilicity. J. Comput. Aided Mol. Des., 1998, 12, 573.

[37] Héberger, K.; Lopata, A. Assessment of nucleophilicity and electrophilicity of radicals, and palr and enthalpy effects on radical addition reactions, J. Org. Chem., 1998, 63, 8646.

[38] Héberger, K.; Görgényi, M. Principal component analysis of Kováts indices for carbonyl compounds in capillary gas chromatography. $J$. Chromatogr. A, 1999, 845, 21.

[39] Damborsky, J.; Berglund, A.; Kuty, M.; Ansorgová, A.; Nagata, M.; Sjöström, M. Mechanism-based quantitative structure-biodegradability relationships for hydrolytic dehalogenation of chloro- and bromoalkenes. Quant. Struct. Act. Relat., 1998, 17, 450.

[40] Zaliani, A.; Gancia, E. MS-WHIM scores for amino acids: A wew 3Ddescription for peptide QSAR and QSPR studies. J. Chem. Inf. Comput. Sci., 1999, 39, 525.

[41] Katritzky, A.R.; Tamm, T.; Wang, Y.; Karelson, M. A unified treatment of solvent properties. J. Chem. Inf. Comput. Sci., 1999, 39, 692.

[42] Harshman, R.A. Foundations of the PARAFAC procedure: Models and conditions for an" explanatory" multi-modal factor analysis. UCLA Working Papers in Phonetics 1970, vol. 16, p. 1.

[43] Bro, R. PARAFAC. Tutorial and applications. Chemom. Intell. Lab. Syst., 1997, 38, 149.

[44] Kiers, H.A L.; Ten Berge, J.M.F.; Bro, R. PARAFAC2: Part I. A direct fitting algorithm for the PARAFAC2 model. J. Chemometr., 1999, 13, 275.

[45] Carroll, J.D.; Chang, J. Analysis of individual differences in multidimensional scaling via an N-way generalization of "EckartYoung" decomposition. Psychometrika, 1970, 35, 283.

[46] Tucker, R.L. Some mathematical notes on three-mode factor analysis. Psychometrika, 1966, 31, 279.

[47] Tucker, R.L. Relations between multidimensional scaling and threemode factor analysis. Psychometrika, 1972, 37, 3 .

[48] Andersson, C.A.; Bro, R. Improving the speed of multi-way algorithms: Part I. Tucker 3. Chemom. Intell. Lab. Syst., 1998, 42, 93.

[49] de Ligny, C.L.; Spajner, M.C.; van Houwelingen, J.C; Weesie, H.M. Three-mode factor analysis of data on retention in normal-phase highperformance liquid chromatography. J. Chromatogr., 1984, 301, 311.

[50] Gemperline, P.J.; Miller, K.H.; West, T.L.; Weinstein, J.E.; Hamilton, J.C.; Bray, J.T. Principal component analysis, trace elements, and blue crab shell disease. Anal. Chem., 1992, 64, 523.

[51] van der Kloot, W.A.; Kronenberg, P.M. External analysis with threemode principal component models. Psychometrika, 1985, 50, 479.

[52] Smilde, A.K.; Wang, Y.; Kowalski, B.R. Theory of medium-rank second-order calibration with restricted-Tucker models. J. Chemometr., 1994, $8,21$.

[53] Morais, H.; Ramos, C.; Forgács, E.; Cserháti, T.; Oliviera, J.; Illés, T. Three-dimensional principal component analysis employed for the study of the $\beta$-glucosidase production of Lentinus edodes strains. Chemom. Intell. Lab. Syst., 2001, 57, 57.

[54] Oros, G.; Ujváry, I. Botanical fungicides: natural and semi-synthetic ceveratrum alkaloids. Pest. Sci., 1999, 55, 253. 